\title{
Slit-lamp management in contact lenses laboratory classes: learning upgrade with monitor visualization of webcam video recordings
}

Justo Arines, Ana Gargallo

Justo Arines, Ana Gargallo, "Slit-lamp management in contact lenses laboratory classes: learning upgrade with monitor visualization of webcam video recordings," Proc. SPIE 9289, 12th Education and Training in Optics and Photonics Conference, 92891H (17 July 2014); doi: 10.1117/12.2070755

Event: 12th Education and Training in Optics and Photonics Conference, 2013, Porto, Portugal 


\title{
Slit-lamp management in contact lenses laboratory classes: learning upgrade with monitor visualization of webcam video recordings
}

\author{
Justo Arines $^{\mathrm{a}^{*}}$ and Ana Gargallo ${ }^{\mathrm{a}}$ \\ ${ }^{a}$ Departamento de Física Aplicada (Área de Óptica) Universidade de Santiago de Compostela, \\ Facultad de Óptica y Optometría (Campus Vida), CP: 15782 Santiago de Compostela, 00555-9642
}

\begin{abstract}
The training in the use of the slit lamp has always been difficult for students of the degree in Optics and Optometry. Instruments with associated cameras helps a lot in this task, they allow teachers to observe and control if the students evaluate the eye health appropriately, correct use errors and show them how to do it with a visual demonstration. However, these devices are more expensive than those that do not have an integrated camera connected to a display unit. With the aim to improve students's skills in the management of slit lamp, we have adapted USB HD webcams (Microsoft Lifecam HD-5000) to the objectives of the slit lamps available in our contact lenses laboratory room. The webcams are connected to a PC running Linux Ubuntu 11.0; therefore that is a low-cost device. Our experience shows that single method has several advantages. It allows us to take pictures with a good quality of different conditions of the eye health; we can record videos of eye evaluation and make demonstrations of the instrument. Besides it increases the interactions between students because they could see what their colleagues are doing and take conscious of the mistakes, helping and correcting each others. It is a useful tool in the practical exam too. We think that the method supports the training in optometry practice and increase the student's confidence without a huge outlay.
\end{abstract}

Keywords: contact lenses practice, slip-lamp, optometry, webcam.

\section{INTRODUCTION}

Slit-lamp, also known as biomicroscope, is one of the most important tools in optometry and ophthalmology practice. It is a low-power microscope that allows the professional to observe live tissues of the eye. Due to its transparency, the eye is the only structure in the body that can be evaluated with a live non-invasive histological exam. The biomicroscope consists of an observation system, which is a binocular microscope; an illumination system, which is a bright light source with a mechanism that allows control the width of the slit; and the mechanical support for its coordination. ${ }^{1}$

The optometrist uses the slit lamp to evaluate health condition of the anterior segment of the eye by focusing the light in an ocular structure with the desired width and observing that structure under an oblique angle. The examiner should develop a routine that is repeated on each patient so that nothing is overlooked and all structures are examined for abnormality. An anterior-to-posterior approach should ensure completeness, and a specific procedure has been suggested. ${ }^{2}$ In addition, the biomicroscope is useful in the assessment of contact lenses fitting and other eye exams as tonometry, gonioscopy, etc. Therefore, it is very important for future professionals in the eye health care to learn the appropriate management of this instrument, which requires a lot of time of training.

In the Degree in Optics and Optometry of the Universidade de Santiago de Compostela the students learn the theoretical principles of the slit lamp as an optometric instrument, beside they have to learn its management in a clinical environment in the contact lenses practical training. That task could be very complicate for them; we have observed that students do not understand very well how the slit lamp works. They have a lot of problems with focusing the slit-lamp in the structure they want to evaluate; especially the cornea and the lens, as well as they have difficulties managing the instrument with easiness and speed. These problems in the learning process generate a loss of confidence in the student that can lead to a discouragement to training classes.

* justo.arines@usc.es; phone 0034881813509

12th Education and Training in Optics and Photonics Conference, edited by

Manuel F. P. C. Martins Costa, Mourad Zghal, Proc. of SPIE Vol. 9289, 92891H

(C) 2014 SPIE, OSA, IEEE, ICO · doi: 10.1117/12.2070755 
In order to improve the slit lamp training of our students we developed a device to help us in the control and evaluation. This device consists of a webcam adapted to the slit lamp available in our contact lenses laboratory rooms, and connected to PC. This is a low cost method and it results very useful in the optometry and contact lenses practical teaching. The visualization at the same time of the eye evaluation conducted by a student allows teachers to correct the mistakes with the focus, the routine in the examination as well as the management of the biomicroscope. Students were also asked in a survey about the satisfaction of the use of webcams in the classes. Our results point that the use of webcams adapted to a slit-lamp, accompanied by a routine in the examination, increase self-confidence and interaction between them.

\section{METHODS}

\subsection{Materials}

We use USB HD webcams with microphone (Microsoft Lifecam HD-5000). The webcams are connected to a PC running Linux Ubuntu 11.0. The slit-lamp used where those placed in the contact lenses laboratory room and there are different models from different trademarks. We develop a device to adapt webcam to one of the slit lamp objectives. That device is a metal tube with the interior surface covered with foam (fig.1a). The webcam is placed inside the tube in one side; the other side is adapted to one ocular with four screws which allow placing the cameras in oculars from different biomicroscopes. (fig. 1b)

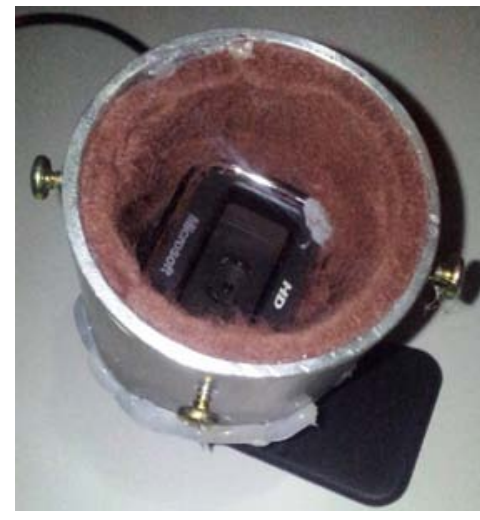

(a)

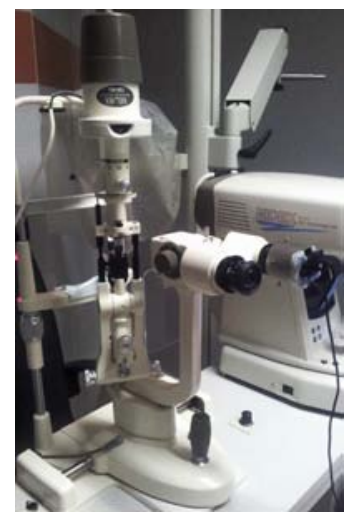

(b)

Fig. 1: (a) Image of the webcam with its adaptor; (b) Image of the slit-lamp with the webcam incorporated on the right hand objective.

\subsection{Routine examination}

We provide students with a routine examination outline. This routine consists in the evaluation of the eye starting with the exam of the eyelids and lashes with diffuse illumination, looking for alterations in lashes, meibomian glands, lids and tear meniscus. Diffuse illumination is used to observe conjunctiva. After conjunctiva they assess cornea and tear film with parallelepiped illumination. After the more anterior structures are observed and noted, the iris and anterior chamber angle can be assessed, followed by examination of the crystalline lens with parallelepiped illumination too.

\subsection{Evaluation of the method}

At the end of the practical period students were evaluated. The exam consisted of a slit-lamp examination of a colleague's eye performed in a maximum of three minutes; we assessed the skill to manage with easiness the biomicroscope, the correctness of the routine and the well-focusing during the eye health examination.

We elaborated a survey with the aim of knowing the opinion of the students about the use of webcams in the slit-lamp training,. They were asked to assess from 1 (nothing) to 5 (very high) the (1) difficulty in the management of the slit- 
lamp, (2) the difficulty compared with other techniques, (3) the reduction in the training time, (4) the utility of webcams during the learning process and (5) the increase in their confidence with the support of webcams. Besides they were asked about reporting the difficulties and advantages in the use of webcams for slit lamp training.

\section{RESULTS AND DISCUSSION}

We have evaluated the use of webcams adapted to a slit-lamp in the contact lenses training classes. We observe that this is a low cost method to help students in the management of the instrument. We use it to make demonstrations where they can see an eye evaluation at the same time that the observer, it is important to make them conscious about what they have to observe and how to do it (see fig. 2 as example of use). This also allows the interaction between students, when one of them is doing an observation of eye health, a colleague can see simultaneously with him and realize of the mistakes in focusing and routine. In the other part, teachers are able to correct focus and management errors during the training period when they are in the contact lenses laboratories. One of the potential uses of webcams is to create an internal network that allows selecting the camera of your election in one PC.

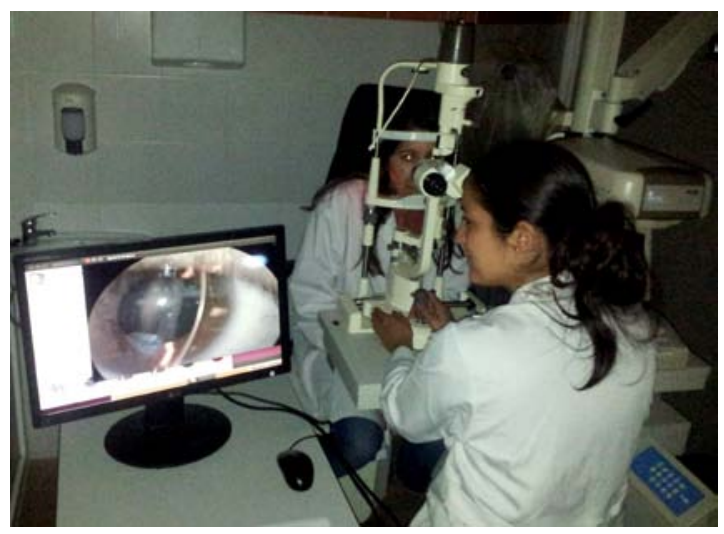

Fig. 2: Picture of a teacher showing how to make the exploration to the students.

At the end of the 4 training sessions, student made a final exam. Most of them were able to do an eye evaluation in 3 minutes, others needed more time. We observed that some had problems in focusing the slit-lamp on the lens and/or cornea; nevertheless, we are satisfied with the test result because we are aware that practice time was not enough in this course, but it will be completed in advanced courses. Students completed the proposed survey at the end of the exam.

Concerning the difficulty of the slit-lamp management, over $80 \%$ of the students found it between medium and high degree of difficulty (figure 3.a). The 62\% considered this technique harder to learn than others (figure 3.b). About $50 \%$ of the students reported that this method reduces the learning period (figure 3.c), moreover a 33\% of students considered that the use of webcams increases a lot their confidence in the management of the slit-lamp and other 35\% pointed a significant increase (figure 3.d). Finally, the $90 \%$ of them found very helpful the use of this method in their training sessions (figure 3.e).

More commented difficulties in the use of the biomicroscope for eye health evaluation were correct focus on the cornea and lens, the control of illumination arm, and the selection of magnification with easiness. They found very helpful the use of webcams for the teacher's explanations concerning the observation techniques and the general management of the slit-lamp. 


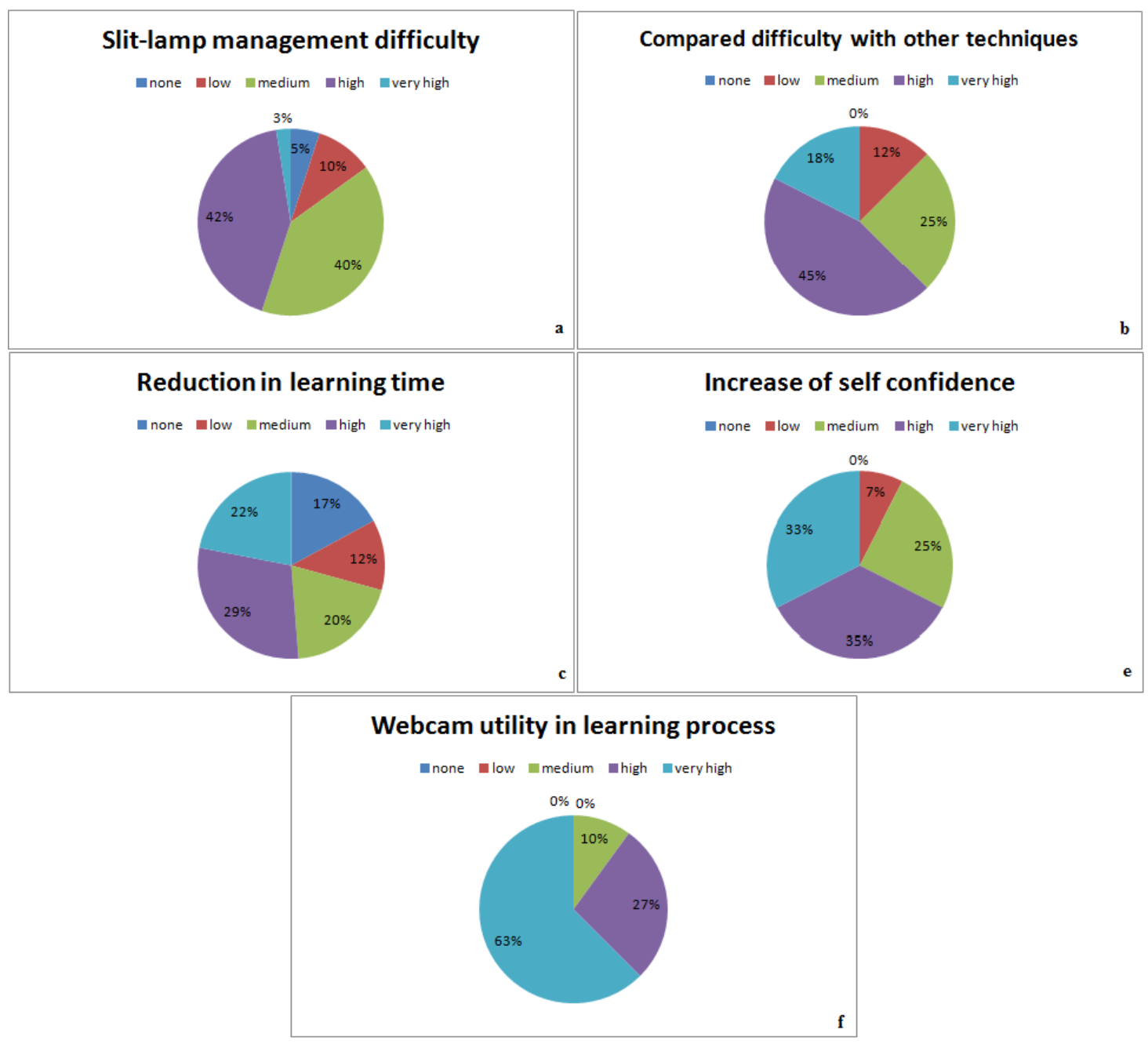

Fig. 3: Results of the survey.

\section{CONCLUSION}

The use of webcams in the slit-lamp training is a low cost method that allows a better understanding of the management and the student evaluation. It contributes to increase the students' self-confidence and the interactions between them and the teacher. The proposed device helps the teacher to show and control the evolution of the student skills related with the management of the slit-lamp. We used the system to show the examination routing, show how students should see the different anatomical structures of the eye (in particular the cornea and posterior and anterior faces of the lens), record videos and pictures with abnormal anatomical findings, follow the improvement in the slit-lamp management of each students, and record examinations in order to show the students they errors. The success achieved makes us to think in other potential uses as recording the outer management of the instrument, creating an internal network for control in a remote PC the observations made in the different examination rooms, and recording and showing the insertion and extraction of contact lenses among others.

\section{REFERENCES}

[1] Grossvenor, T., [Primary Care Optometry] $5^{\text {th }}$ edition, Butterworth Heinemann Elsevier, 137-182 (2007).

[2] Benjamin, W.J., [Borish's Clinical Refraction] $2^{\text {nd }}$ edition, Butterworth Heinemann Elsevier, 485-510 (2006). 